\title{
Nephrotic-range proteinuria in a patient with dengue fever: a case report from Bangladesh
}

\author{
Shapur Ikhtaire \\ Department of Internal Medicine, Bangabandhu Sheikh Mujib Medical University (BSMMU), \\ Shahbag, Dhaka, Bangladesh
}

\begin{abstract}
We report a case of nephrotic range proteinuria with 24-hour urine protein level of $18.3 \mathrm{~g} / \mathrm{day}$, which developed following dengue fever (DF). The patient did not exhibit classical features of nephrotic syndrome (NS) and his renal function was not compromised during his illness. Proteinuria resolved without any specific treatment and precluded renal biopsy. Though the dengue fever and associated renal disorders are self-limiting, renal involvement in severe dengue infection is growingly seen in recent years and could cause increased mortality and long-term morbidity.
\end{abstract}

IMC J Med Sci 2018; 12(2): 86-89. EPub date: 06 September 2018

\section{Introduction}

Dengue fever is an acute febrile illness accompanyied by constitutional symptoms. Dengue haemorrhagic fever (DHF) and dengue shock syndrome (DSS) are the severe forms of dengue infection, which have become a major international public health issue [1]. Expanded dengue syndrome (EDS) is a relatively new entity which incorporates a wide spectrum of unusual manifestations of dengue virus infection involving kidney, liver, brain, heart and muscle [1]. The dengue virus is an RNA arbovirus from the genus Flavi virus (family Flaviviridae). It has four closely related serotypes: DEN-1, DEN-2, DEN-3 and DEN-4 based on antigenic characteristics of dengue virus. More recently, a new serotype DEN-5 was identified, which caused an epidemic of dengue in Malaysia in 2007 [2,3]. Over the past three decades, there has been a global increase in the frequency and epidemics of DF, DHF, DSS with a concurrent rise in disease incidence [1]. Though it is a self-limiting disease, in the recent years complications involving specific organ systems and dengue related mortality and morbidity have been observed at an increasing rate [4-6]. Here, we describe a case of dengue virus infection who exhibited massive nephrotic range proteinuria (18.3 g/day) following DF. Proteinuria improved without any specific treatments and precluded renal biopsy. To date, proteinuria of $18.3 \mathrm{~g} /$ day in DF has never been reported from Bangladesh and rarely from the other parts of the world.

\section{Case report}

A 17-year-old boy without any past medical illness presented through emergency department of a hospital in Dhaka city with a 3-day history of high grade fever, chills, myalgia and headache. On admission, he was febrile $\left(102^{0} \mathrm{~F}\right)$, with congested eyes without any systemic signs and bleeding manifestations. His pulse was 100 beats/min and blood pressure was $120 / 85 \mathrm{~mm}$ of $\mathrm{Hg}$. Initial investigation on admission showed hemoglobin 116 $\mathrm{gm} / \mathrm{L}$, total white blood cell (WBC) $7.6 \times 10^{9} / \mathrm{L}$, platelet count $155 \times 10^{9} / \mathrm{L}$, which dropped to $110 \times 10^{9} / \mathrm{L}$ the next day. Packed cell volume (PCV) $35 \%$, erythrocyte sedimentation rate (ESR) $05 \mathrm{~mm}$ in first hour and Dengue NS-1 Ag was positive $\left(3^{\text {rd }}\right.$ day of his fever). His liver transaminase, renal function, blood urea and electrolytes were within normal limit. The case was diagnosed as DF and treated symptomatically with adequate fluid and

\section{Address for Correspondence:}

Dr. Shapur Ikhtaire, Department of Internal Medicine, Bangabandhu Sheikh Mujib Medical University (BSMMU); Email: ikhtaireshapur@yahoo.com 
anti-pyretic (paracetamol). By day 5 of fever, he noticed excessive frothiness of urine and he developed mild puffiness of face without any oedema, ascites, plural effusion and rise of blood pressure. Immediate urine examination revealed, presence of proteinuria $(+++)$, RBC 2-3/HPF and pus cell 6-8/HPF. This proteinuria was quantified by 24 hours urinary total protein (UTP) test, which surprisingly exhibited as UTP $18.3 \mathrm{gm} / 24$ hours (UTV $5000 \mathrm{ml}$ ). Accordingly, other relevant tests were done to exclude other potential systemic causes of nephrotic range proteinuria and preexisting glomerulonephritis (GN). These included urine phase contrast microscopy to delineate glomerular pathology, which showed RBC 6-8/ HPF, dysmorphic RBC-15\%, pus cell 8-10/HPF without the presence of any cast. Urine and blood culture revealed no growth. Anti-nuclear antibody (ANA), Anti ds-DNA, cytoplasmic antineutrophil cytoplasmic antibodies (c-ANCA), perinuclear antineutrophil cytoplasmic antibody (p-ANCA), C3, $\mathrm{C} 4$, immunochromatograpic test for malaria and hepatitis B surface antigen (HBsAg) were found negative. Serum creatinine and albumin were within normal reference limit. Surprisingly, regardless of massive nephrotic range proteinuria the patient did not have the essential criteria of classical nephrotic syndrome of hypoalbuminaemia, dyslipidaemia and oedema. By the $7^{\text {th }}$ day of his illness, he noticed yellow coloration of eyes, severe myalgia and gum bleeding. At this stage his serum bilirubin was 37.62 $\mu \mathrm{mol} / \mathrm{L}$ (normal 3-22 $\mu \mathrm{mol} / \mathrm{L}$ ) AST $246 \mathrm{U} / \mathrm{L}$ (normal-15-37 U/L), CPK 667 U/L (normal-24-190 U/L). Therefore, along with his initial presentation, additionally he developed hepatitis and myositis. Although, he had no bleeding manifestation, relevant tests were done which included bleeding and clotting time (BT, CT), reticulocyte count, activated partial thromboplastin time (APTT), INR, direct and indirect Coomb's tests. All of them were within normal limit. His platelet count was $125 \times 10^{9} / \mathrm{L}$ and peripheral blood film (PBF) showed normocytic normochromic anaemia without any features of haemolysis. Anti-dengue $\operatorname{IgG}$ was positive while IgM was negative. During his spectrum of illness, he did not exhibit any significant bleeding manifestation or any features of plasma leakage like ascites, plural effusion and rise of hematocrit value. His chest x-ray was normal and ultra sonogram of abdomen revealed mild splenomegaly.
Based on clinical features and laboratory investigation he was diagnosed as a case of expanded dengue syndrome with unusual manifestation of nephrotic range proteinuria along with hepatitis and myositis.

By day 10 of his illness, he was afebrile, regained some well-being. His jaundice, myalgia and proteinuria began to subside. Renal biopsy was not done as the proteinuria was self-resolving. He was treated with low dose ACEI ( $25 \mathrm{mg} /$ day) only. No glucocorticoid was given. Follow up at 2 weeks and 2 months showed remarkable resolution of proteinuria with level of $0.62 \mathrm{~g} /$ day and $0.15 \mathrm{~g} /$ day (within normal limit) respectively. His complete blood count, serum transaminases, creatinine and routine urine examination were within normal limit. No renal biopsy was done subsequently due to selfresolution of proteinuria.

\section{Discussion}

Dengue fever, a significant public health problem in many countries of the world, is an acute febrile illness accompanied by constitutional symptoms. Clinical manifestations of dengue are variable and the spectrum ranges from asymptomatic to undifferentiated fever to severe dengue or multi-organ failure. Several reports have described dengue related morbidity and mortality due to complications involving gastrointestinal, hepatic, renal, neurological and respiratory systems [3-8].

Renal manifestation associated with dengue infection is discussed in the context of the present case. Dengue infection has been related with range of renal disorders like acute renal failure, haematuria, proteinuria, GN and has been observed during or sharply after acute dengue infection [9]. Proteinuria and abnormal urinary sediments were the most frequently seen renal abnormalities of dengue infection [10,11]. Proteinuria and hematuria were observed in $74 \%$ and $31 \%$ of hospitalized dengue patients respectively. Proteinuria was transient and self-limiting in majority of cases $[9,12]$. However, nephrotic range proteinuria was observed in two cases with DHF [12], and another study reported massive proteinuria of $335.7 \mathrm{mg} / \mathrm{kg} /$ day following DHF [13]. Unlike our patient, significant proteinuria was strongly associated with DHF compared to DF alone [12-14]. Different types of GN have been 
reported during or sharply after dengue infection. Study reported microscopic haematuria in $18 \%$ and $27 \%$ of patients with DF and DHF respectively [15]. Several studies have reported immune complex mediated injury to the glomerulus in patients with dengue infection. Mesangial proliferation and immune complex deposition were the most dominant histological features [16]. Exploration of renal biopsy materials from the patients of severe dengue infection with kidney injury have revealed deposition of $\mathrm{IgG}, \operatorname{IgM}$, and $\mathrm{C} 3$ in the glomeruli and thickening of the basement membrane with hypertrophy of the mesangial cells. However, the extent of proteinuria was found concurrent with the severity of thrombocytopenia and normalization of platelet count was observed during resolving state of proteinuria $[3,17]$. Acute kidney injury (AKI) is a potential complication of severe dengue infection, and tied to multi-organ failure and increased mortality. The incidence of AKI in patients with DHF is variable and spectrum ranges from raised serum creatinine, proteinuria, GN, haemolysis, hypotension and rhabdomyolysis [3,18]. DSS, DHF, diabetes mellitus, nephrotoxic drug, concurrent bacteremia, chronic kidney diseases, old age and gastrointestinal bleeding were associated with a higher risk of AKI following dengue infection $[19,20]$.

Our patient was diagnosed as a case of EDS having nephrotic-range proteinuria of $18.3 \mathrm{~g} /$ day with hepatitis and myositis. To date, this extent of proteinuria following DF has never been reported from our country and reported rarely from the other parts of the world as well. A few cases of nephrotic range proteinuria in dengue infection had been reported earlier where up to $10.8 \mathrm{~g}$ /day to 335.7 $\mathrm{mg} / \mathrm{kg} /$ day proteinuria was observed $[10,13]$. Unlike our patient, massive proteinuria has been found to be strongly associated with DHF compared to classical DF and severe thrombocytopenia [14,17]. Surprisingly, even though our case had massive proteinuria (18.3 g/day), the patient did not have the features of classical nephritic syndrome as with few other reported cases earlier [17]. In other reported cases with nephrotic range proteinuria following dengue infection, renal biopsy was not done, as spontaneous resolution of proteinuria occurred with remission of dengue infection $[12,13,17]$. In our patient, proteinuria also resolved to normal reference limit $(<0.15 \mathrm{~g} /$ day $)$ in subsequent follow up without any specific treatment. Therefore, renal biopsy was not done. Conversely, dengue infection may be associated with systemic diseases like IgA nephropathy and SLE in rare occasion [9,21]. However, one patient had p-ANCA associated myeloperoxidase (MPO) specific anti-glomerular basement membrane (GBM) disease and renal biopsy of the same patient revealed severe crescentic glomerulonephritis [16]. Considering these, our patient was advised to seek medical attention if he noticed any urinary problems in future. This case is important as renal involvement in dengue infection is often poorly addressed and could potentially increase the mortality and morbidity. However, well-designed and large prospective studies are required to understand the pathophysiology of massive proteinuria in dengue infection. This could improve the outcome of the dengue infection and its complications.

\section{Acknowledgement}

I gratefully acknowledge the authorities of Central Hospital Ltd, Dhanmondi, Dhaka, for kindly permitting to publish the case. I am thankful for the support of indoor medical officers of the medicine unit of the hospital for the management and followup of the case.

\section{Author's contribution}

SI was responsible for diagnosis, management of the case and manuscript writing.

\section{Competing interest}

The author declared no competing interests.

\section{Funding}

None

\section{References}

1. World Health Organization. Comprehensive guidelines for prevention and control of dengue and dengue haemorrhagic fever. New Delhi, India: World Health Organization; 2011. p 212 SEARO Technical Publication Series No.: 60 
2. Normile D. Tropical medicine. Surprising new dengue virus throws a spanner in disease control efforts. Science. 2013; 342(6157): 415. doi: 10.1126/science.342.6157.415

3. Oliveira JF, Burdmann EA. Dengue-associated acute kidney injury. Clin Kidney J. 2015; 8(6): 681-5.

4. Gurugama $\mathrm{P}$, Jayarajah U, Wanigasuriya $\mathrm{K}$, Wijewickrama A, Perera J, Seneviratne SL. Renal manifestations of dengue virus infections. J Clin Virol. 2018; 101: 1-6.

5. Seneviratne SL, Malavige GN, de Silva HJ, Pathogenesis of liver involvement during dengue viral infections. Trans. R. Soc. Trop. Med. Hyg. 2006; 100(7): 608-14.

6. Mathew S, Pandian JD. Stroke in patients with dengue. J Stroke Cerebrovasc Dis. 2010; 19(3): 253-6.

7. Laoprasopwattana $\mathrm{K}$, Pruekprasert $\mathrm{P}$, Dissaneewate P, Geater A, Vachvanichsanong P. Outcome of dengue hemorrhagic fevercaused acute kidney injury in Thai children. J. Pediatr. 2010; 157(2): 303-9.

8. Khan E, Kisat M, Khan N, Nasir A, Ayub S, Hasan R. Demographic and clinical features of dengue fever in Pakistan from 2003-2007: a retrospective cross-sectional study. PloS One. 2010; 5(9): e12505.

9. Lizarraga KJ, Nayer A. Dengue-associated kidney disease. J Nephropathol. 2014; 3(2): 57-62.

10. Hanna JN, Ritchie SA, Phillips DA, Serafin IL, Hills SL, van den Hurk AF, Pyke AT, McBride WJ, Amadio MG, Spark RL. An epidemic of dengue 3 in far north Queensland, 1997-1999. Med J Aust. 2001; 174(4): 178-82.

11. Barsoum RS, Francis MR, Sitprija V. Renal involvement in tropical diseases, Atlas of Diseases of the Kidney, Current Medicine Inc. Philadelphia, PA. 1999; 4: 6.18-6.19.

12. Vasanwala FF, Puvanendran R, Ng JM, Suhail SM. Two cases of self-limiting nephropathies secondary to dengue haemorrhagic fever. Singapore Med J. 2009; 50(7): e253-5.

13. Hutspardol S, Prommalikit O, Upiya N, Chataroopwijit J, Khemakanok K, Assadamongkol K. Heavy proteinuria following dengue hemorrhagic fever. Southeast Asian J Trop Med Public Health. 2011; 42(3): 579-82.

14. Vasanwala FF, Thein TL, Leo YS, Gan VC, Hao Y, Lee LK, et al. Predictive value of proteinuria in adult dengue severity. PLoS Negl Trop Dis. 2014; 8: e2712.

15. Wenderfer SE. Viral-associated glomerulopathies in children. Pediatr Nephrol 2015; 30: 1929-38.

16. Lizarraga KJ, Florindez JA, Daftarian $P$, Andrews DM, Ortega LM, Mendoza JM, Contreras GN, Nayer A. Anti-GBM disease and ANCA during dengue infection. Clin Nephrol. 2015; 83(2): 104-10.

17. Hebbal P, Darwich Y, Fong J, Hagmann SHF, Purswani MU. Nephrotic-range proteinuria in an eight-year-old traveler with severe dengue: Case report and review of the literature. Travel Med Infect Dis. 2016; 14(1): 45-8.

18. Aroor S, Kumar S, Mundkur S, Kumar M. Hemolytic uremic syndrome associated with Dengue fever in an adolescent girl. Indian $J$ Pediatr. 2014; 81(12): 1397-8.

19. Wei HY, Shu PY, Hung MN. Characteristics and Risk Factors for Fatality in Patients with Dengue Hemorrhagic Fever, Taiwan, 2014. Am J Trop Med Hyg. 2016; 95(2): 322-7.

20. Lee IK, Liu JW, Yang KD. Clinical and laboratory characteristics and risk factors for fatality in elderly patients with dengue hemorrhagic fever. Am J Trop Med Hyg. 2008; 79(2): 149-53.

21. Upadhaya BK, Sharma A, Khaira A, Dinda AK, Agar- wal SK, Tiwari SC. Transient IgA nephropathy with acute kidney injury in a patient with dengue fever. Saudi J Kidney Dis Transpl. 2010; 21(3): 521-5. 